\title{
High-Frequency Conductivity of Optically Excited Charge Carriers in Hydrogenated Nanocrystalline Silicon Investigated by Spectroscopic Femtosecond Pump-Probe Reflectivity Measurements
}

\author{
Wei He ${ }^{\mathrm{a}}$, Igor V. Yurkevich ${ }^{\mathrm{b}}$, Ammar Zakar ${ }^{\mathrm{a}}$, Andrey Kaplan ${ }^{\mathrm{a}, *}$ \\ ${ }^{a}$ University of Birmingham, School of Physics and Astronomy, Birmingham, UK, B15 2TT \\ ${ }^{b}$ Aston University, Nonlinearity and Complexity Research Group, Birmingham, UK, B4 \\ $7 E T$
}

\begin{abstract}
We report an investigation into the high-frequency conductivity of optically excited charge carriers far from equilibrium with the lattice. The investigated samples consist of hydrogenated nanocrystalline silicon films grown on a thin film of silicon oxide on top of a silicon substrate. For the investigation, we used an optical femtosecond pump-probe setup to measure the reflectance change of a probe beam. The pump beam ranged between 580 and $820 \mathrm{~nm}$, whereas the probe wavelength spanned 770 to $810 \mathrm{~nm}$. The pump fluence was fixed at $0.6 \mathrm{~mJ} / \mathrm{cm}^{2}$. We show that at a fixed delay time of $300 \mathrm{fs}$, the conductivity of the excited electron-hole plasma is described well by a classical conductivity model of a hot charge carrier gas found at Maxwell-Boltzmann distribution, while Fermi- Dirac statics is not suitable. This is corroborated by values retrieved from pump-probe reflectance measurements of the conductivity and its dependence on the excitation wavelength and carrier temperature. The conductivity decreases monotonically as a function of the excitation wavelength, as expected for a nondegenerate charge carrier gas.
\end{abstract}

Keywords: nanomaterials, high-frequency conductivity, nanosilicon, ultrafast spectroscopy

\footnotetext{
${ }^{*}$ Corresponding author

Email address: a.kaplan.1@bham.ac.uk (Andrey Kaplan)
} 
2014 MSC: 00-01, 99-00

\section{Introduction}

In recent decades, hydrogenated nanocrystalline silicon (nc-Si:H), which is composed of an amorphous silicon phase with embedded nanoclusters of crystalline silicon, has attracted considerable research attention [1, 2]. This material 5 revealed unique electro-optical properties, which have already been investigated for application in photovoltaic solar cells [3], nonvolatile memory devices [4, and thin-film transistors [5]. However, the high-frequency conductivity is not yet well understood or researched, although it the most critical property governing the performance of electro-optical devices. Several critical questions await understanding and explanation: what happens to the charge carriers after they absorb a photon; what is the mechanism of their relaxation and the excess energy release; what physics governs their conductivity. This work tries to address the last question.

In this paper, we report a first attempt to understand the high-frequency 15 conductivity as a function of the pump photon energy. The idea is to establish the dependence of the conductivity immediately (a few hundred femtoseconds) after excitation on the excess energy provided by the photons to the carriers. This short time is sufficient to allow the carrier subsystem to thermalize and settle at a certain thermodynamic distribution characterized by a temperature proportional to the excess energy, but still too short for it to exchange energy with another subsystem of lattice ions.

One of the most advanced tools currently available for the investigation of processes on extremely short timescales is ultrafast optical pump-probe spectroscopy [6]. In brief, the absorption of pump photons with an energy greater 25 than the band gap energy of a semiconductor material generates electron-hole pairs. At sufficiently high concentrations, these charges can be treated as an ambipolar plasma. This free carrier plasma alters the dielectric function of materials and changes the optical response, such as the reflectance, transmittance, 
and polarizability [7, 8, 9]. Thus, investigation of the optical response allows indirect access to the properties of the excited carriers forming the plasma, such as the relaxation and recombination times, concentration, carrier temperature, and scattering mechanism. The pump-probe reflectance measurement is among the most widely used because of its straightforward manageability.

In our investigation, we recorded 25 pump-probe spectra, each of which corresponds to a pump wavelength between 580 and $820 \mathrm{~nm}$. The reflectance is probed by a broadband probe in the range between 765 and $815 \mathrm{~nm}$. The results are analyzed using the Drude conductivity and Boltzmann transport theories. We show that the thermal distribution of the nascent carriers can be described as that of a classical hot nondegenerate gas. The conductivity of the

40 gas monotonically decreases as the excess energy provided by the pump photons decreases.

\section{Experimental}

\subsection{Sample and characterization}



Figure 1: SEM image of cross section of nc-Si:H sample; the top $480 \mathrm{~nm}$-thick layer is nc-Si:H, which was grown on $\mathrm{a} \sim 188 \mathrm{~nm}$ silicon oxide film $\left(\mathrm{SiO}_{2}\right)$ on top of a bulk crystalline silicon substrate.

The investigated samples consist of a layer of hydrogenated amorphous sili45 con $(\alpha$-Si:H) containing nc-Si grown by a modified plasma deposition technique similar to that described elsewhere $[3,10$. The dimensions of the sample layers 
were estimated using cross-sectional scanning electron microscopy (SEM) images. Figure 1 shows that the top layer of the nc-Si:H film is around $480 \mathrm{~nm}$ thick, whereas the underlying silicon oxide film is $\sim 188 \mathrm{~nm}$ thick. The bottom part of the cross section is a bulk crystalline silicon substrate.

The composition of the nc-Si:H film was investigated by Raman spectroscopy and analysis of the transverse optical modes [11. In Figure 2(a), the experimental Raman data are shown as black circles. Two Gaussian functions with centers around 520 and $480 \mathrm{~cm}^{-1}$ were applied to fit the Raman data. The integrated intensity ratio, $I_{520 \mathrm{~cm}^{-1}} /\left(I_{520 \mathrm{~cm}^{-1}}+I_{480 \mathrm{~cm}^{-1}}\right)$, can be used to evaluate the crystalline volume fraction in nc-Si:H films [12, where $I_{520 \mathrm{~cm}^{-1}}$ and $I_{480 \mathrm{~cm}^{-1}}$ represent the areas of the respective Gaussians. The fitting results are shown as a red solid line in Figure 2(a). The volume fraction of the silicon crystalline phase estimated using this procedure is around $35 \%$, and the rest is amorphous silicon.

The X-ray diffraction (XRD) data shown in Figure2(b) were used to estimate the size of the embedded nanoclusters [13, 14, 15. Using the Scherrer equation [16, 17, we evaluated the mean diameter as $\langle a\rangle \approx 6 \mathrm{~nm}$. The equation relates the broadening of a peak in a diffraction pattern to the size of the embedded clusters.
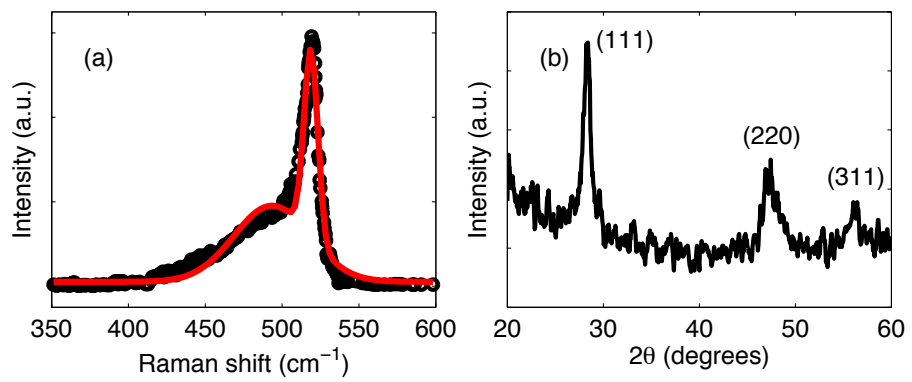

Figure 2: (a) Experimental Raman data (black circles) of the nc-Si:H film. Red line shows a fit with two Gaussians. (b) XRD measurement showing three broadened diffraction peaks: (111), (220), (311). 


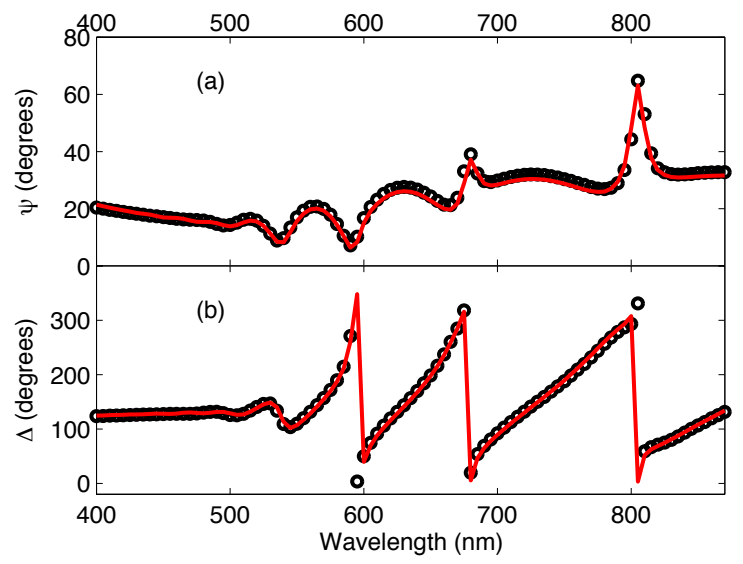

Figure 3: Measured (a) $\psi(\lambda)$ and (b) $\Delta(\lambda)$ (black circles) obtained at an incident angle of $70^{\circ}$. Red solid lines show simulations of multilayer optical model.

\subsubsection{Determination of the dielectric function}

To obtain the optical properties of the nc-Si:H film, we used a Woollam commercial spectroscopic ellipsometer to measure the polarization state amplitude ratio $\psi(\lambda)$ and the phase difference $\Delta(\lambda)$ at an incident angle of $70^{\circ}$ and over a spectral range of 400 to $870 \mathrm{~nm}$. Figure 3 shows the experimental data as black circles. To fit the experimental results and to determine the dielectric function of the top nc-Si film, we used the classical Lorentz model and four-term Forouhi-Bloomer model 18 to express the dispersion relations of the underlying $\mathrm{SiO}_{2}$ film and crystalline silicon bulk, respectively. To describe the amorphous silicon phase of the nc-Si film, a one-term Forouhi-Bloomer model 19] was applied. Because the nc-Si:H layer consists of an amorphous phase and a crystalline phase, the Bruggeman effective medium approximation was used to express the effective dielectric function [20, 21]. Finally, by using the transfer matrix method [22, 23, the multilayer optical mode was constructed to simulate the ellipsometric response. We also included in the multilayer optical model a thin layer of $\sim 10 \mathrm{~nm}$-thick $\mathrm{SiO}_{2}$ to account for the native oxide on the surface of the top layer. The thicknesses of the layers in the optical model were taken from the SEM cross section measurements. The red solid lines in 
Figure 3 show the results of the simulation. The real and imaginary parts of the effective dielectric function of the nc-Si:H layer, $\epsilon_{\text {eff }}^{0}$, are shown in Figure 4 The obtained dielectric function was used later to analyze the results of the pump-probe measurements.



Figure 4: Effective dielectric function of the nc-Si:H layer, $\epsilon_{\text {eff }}^{0}=\Re\left(\epsilon_{\text {eff }}^{0}\right)+i \cdot \Im\left(\epsilon_{\text {eff }}^{0}\right)$, obtained from measurements shown in Fig. 3 and simulations of the multilayer optical model. Left and right axes correspond to the real and imaginary parts of the complex dielectric function, respectively. Inset: An enlarged portion of the spectra between 750 and $825 \mathrm{~nm}$.

\subsection{Pump-probe setup}

An ultrafast laser system (Coherent) was used in the pump-probe setup; it delivers $1 \mathrm{kHz}, 50 \mathrm{fs}$ laser pulses centered around $790 \mathrm{~nm}$. The main beam is split into high-intensity pump pulses and low-intensity probe pulses by a pellicle beam splitter. Using a light frequency converter (OPA), the pump wavelength can be converted to any wavelength between 580 and $820 \mathrm{~nm}$. The pump fluence was fixed at $0.6 \mathrm{~mJ} / \mathrm{cm}^{2}$, and the probe intensity of the fundamental beam frequency was further attenuated by a neutral density filter. The incident angle of the probe beam was set to $70^{\circ}$, whereas the angle of the pump was set to around $50^{\circ}$. The polarization of the probe beam was adjusted to provide equal contributions of the $s$ - and $p$-polarization components, and the pump beam was always in the $s$-polarized state. The spatial overlap between the probe and pump spots on the sample surface was checked by a CCD camera equipped with a magnifying lens. The temporal overlap between the probe 
and pump pulses was checked by detecting the sum frequency generation on a BBO crystal. By using a computer-controlled retroreflector, the probe delay time with respect to the pump was fixed at $300 \mathrm{fs}$. Finally, the wavelength of the reflected probe beam was analyzed by a spectrometer (Ocean Optics QE65 Pro), and the collected data are presented here as $\Delta R(\lambda) / R_{0}$, where $R_{0}$ is the reflectance without pumping. Further, $\Delta R=R(t)-R_{0}$ is the change in reflectance induced by the pump excitation $[24,25$, where $R(t)$ is the reflectance measured at a certain delay time (300 $f s$ in this work) by the probe. More details on the pump-probe setup and data modeling are available elsewhere [23, 26, 27. The $300 f s$ delay time was chosen because it is sufficiently long for the excited free carrier plasma to build up in the nc-Si:H layer but still short enough to avoid significant population decay [23, 28] and carrier-lattice interactions [29].

\section{Experimental Results, Data Analysis, and Discussion}

The application of the pump pulse generates a free carrier plasma inside the nc-Si:H layer. These excited carriers change the effective dielectric function of the nc-Si:H layer and the detected intensity of the reflected probe. The excited carrier concentration can generally be derived from the measured intensity change of the reflected probe. The relation between the concentration and the reflectance can be obtained using the classical Drude model 24, 25, 30. According to the Drude model, the dielectric function of the excited layer after pump excitation, $\epsilon_{e f f}$, can be approximated as follows:

$$
\epsilon_{e f f}=\epsilon_{e f f}^{0}-\frac{\omega_{p}^{2}}{\omega^{2}+i \cdot \Gamma \omega}
$$

where $\omega$ is the probing frequency. Further, $\omega_{p}$ is the plasma frequency, which is given as $\omega_{p}^{2}=\frac{e^{2} N_{e h}}{\epsilon_{0} m^{*} m_{e}}$, where $e, N_{e h}, m^{*}$, and $m_{e}$ are the electron charge, carrier concentration, optical mass, and free electron mass, respectively. An optical mass of $m^{*}=0.17$ was recently estimated for this material [23]. Thus, the excited carrier concentration $N_{e h}$ can be deduced from $\omega_{p}$. However, there is another important factor affecting the conductivity, that is, the scattering 
rate $\Gamma$. Thus, the main task is to obtain $\Gamma$ and $N_{e h}$ for each pump spectrum and establish the high-frequency conductivity according to the relation $\sigma_{0}=$ $e^{2} N_{e h} /\left(\Gamma m^{*} m_{e}\right)$. Note that at a relatively short delay time of around $300 \mathrm{fs}$, carrier-carrier collisions are the main scattering process contributing to $\Gamma$. The carrier-phonon collision contribution to the scattering can be neglected, as it is realized on much longer timescales.

Simultaneous experimental determination of $\Gamma$ and $N_{e h}$ is a complex task that generally requires at least two independent measurements, such as a combination of the transmittance and reflectance [26, or determination of different polarization states of the probe. However, this task can be simplified when $\Gamma$ has a known dependence on $N_{e h}$. Here we refer to known models of carrier-carrier scattering published elsewhere [31. Briefly, these models allow us to calculate the scattering rate as a function of the carrier concentration and carrier temperature as a parameter for the Maxwell-Boltzmann (MB) and Fermi-Dirac (FD) distributions. In this work, we assumed the MB distribution. We note that we independently verified that the FD distribution provides unsatisfactory results. According to the model, the scattering rate can be estimated as follows:

$$
\hbar \Gamma=k_{B} T_{r y d}\left(\frac{T_{n}}{T}\right)^{3 / 2}\left(\ln \frac{T^{2}}{T_{n}^{3 / 2} T_{r y d}^{1 / 2}}\right) M,
$$

where $k_{B}$ is the Boltzmann constant; $T_{n}$ is the Fermi temperature, which is directly related to the carrier concentration as $k_{B} T_{n}=\frac{\hbar^{2}}{2 m^{*} m_{e}}\left(3 \pi^{2} N_{e h}\right)^{2 / 3}$; and $T_{r y d}$ and $M$ are the Rydberg temperature and band structure factor, respectively. The band structure factor can be approximated as close to that of bulk silicon 31. The value of the Rydberg temperature is less well known because of the enhanced carrier-carrier interaction at high carrier concentrations [23]; in our calculation procedure, it was left as an unknown but fixed parameter (that is, it is the same for all pump and probe wavelengths). Finally, for the MB distribution, the temperature of the nascent carriers can be evaluated according

155 to the equipartition theorem, $\frac{3}{2} k_{B} T=\frac{\hbar \omega_{p u m p}-E_{g}}{2}$, where $\hbar \omega_{\text {pump }}$ and $E_{g}$ are the pump photon energy and energy gap of the material, respectively. The latter in our experiment was about $1.2 \mathrm{eV}$, whereas the former was varied. Thus, 
in our fitting procedure we simulate 25 reflectance pump-probe spectra taken at different pump photon frequencies $\omega_{\text {pump }}$ (corresponding to different carrier temperatures $T$ ) for which the carrier concentration $N_{e h}$ is an unknown free parameter, and the Rydberg temperature $T_{r y d}$ is an unknown fixed parameter. The confidence of the simulation procedure is ensured by the small number of fitting parameters and their independence of the probing wavelength in each particular spectrum.

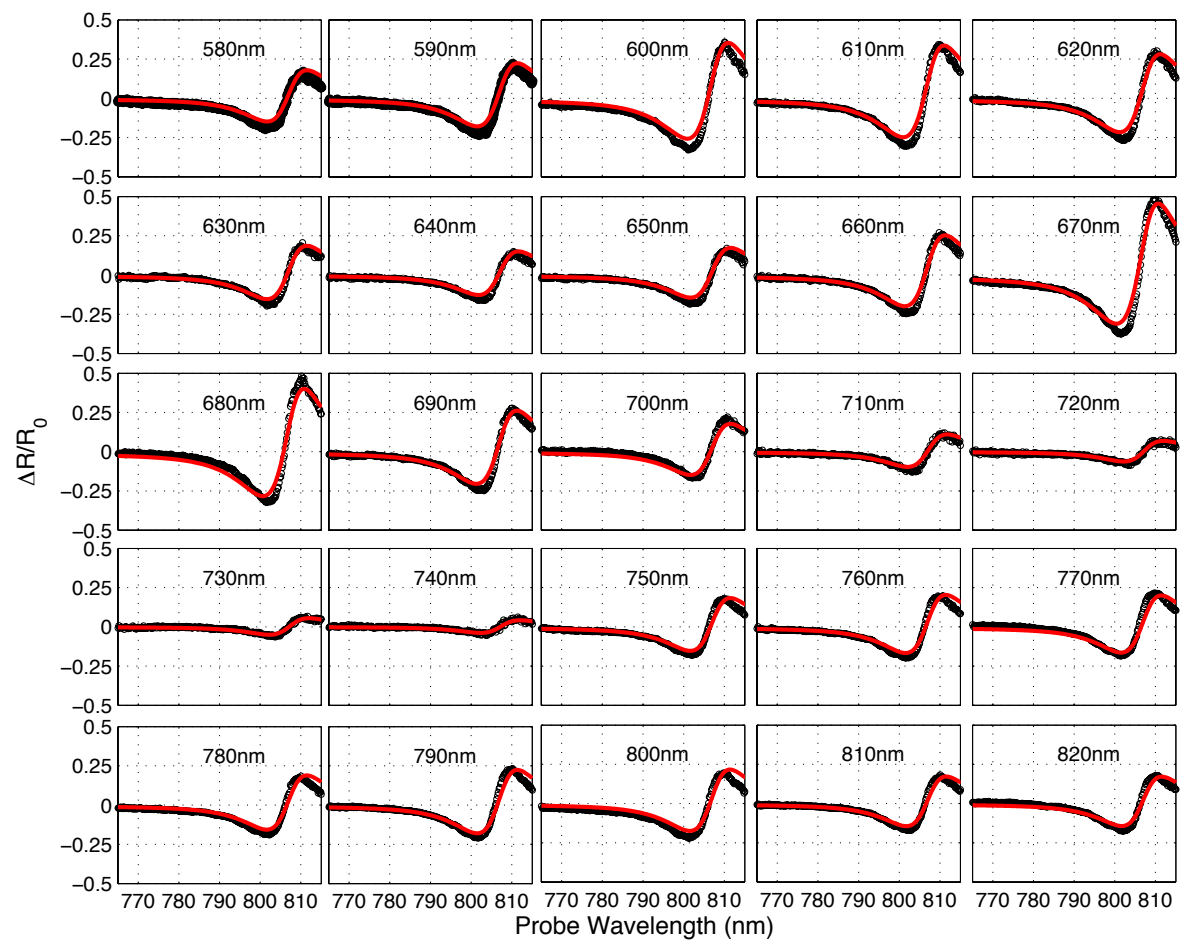

Figure 5: Transient pump-probe experimental data (black dots) for $\Delta R / R_{0}$ as a function of the probe wavelength taken at different pump wavelengths. Red lines denote simulated results.

Figure 5 shows the pump-probe change in the reflectance data, $\Delta R / R_{0}$, as a function of the probe wavelength taken at different pump wavelengths. The probe spectrum spans 770 to $810 \mathrm{~nm}$, whereas the pump wavelength covers the region from 580 to $820 \mathrm{~nm}$ at $10 \mathrm{~nm}$ intervals. All the spectra show a 
Fabry-Perot fringe on the red side, similar to that observed elsewhere [23, 30]. This fringe originates from the interference effect induced by the change in the dielectric function of the layer following excitation by the pump. However, the contrast of the fringe varies with the pump. This clearly indicates that the excitation efficiency and carrier concentration depend on the pump wavelength for this system. This effect has, however, a simple explanation: The absorbance of the pump pulse depends on the conditions of constructive and destructive interference of the incoming and internally reflected light components within the layer.

Using Eqs. 1 and 2 in the multilayer optical model discussed in previous sections, the data $\Delta R / R_{0}$ can be fitted to retrieve $N_{e h}$ and $\Gamma$ as functions of the pump wavelength. The fitting results are shown as red solid lines in Figure 5, which coincide well with the black data points. The retrieved $N_{e h}$ and $\Gamma$ are shown in Figure 6(a) and (b), respectively. The Rydberg temperature used in the calculation, given by $m_{e} m^{*} e^{4} / 4 \pi \hbar^{2}\left(\epsilon \epsilon_{0}\right)^{2}$, was calculated with the standard constants and optical mass given above, except for $\epsilon$, which is a fixed fitting parameter here. The dielectric constant indicates screening of the carrier-carrier interaction. Unlike the $\epsilon$ value for silicon bulk material, which lies between 12 and 13, the value producing the best fit is somewhat lower than 2, demonstrating a tenfold carrier-carrier interaction enhancement, in good agreement with a previous work 23] (please note that in the previous work the scattering rate estimate provided a higher value because the pumped carriers density was higher). The $N_{e h}$ and $\Gamma$ retrieved from the simulation show synchronous oscillation as a function of the pump wavelength. The oscillations originate in the constructive and destructive interference of the multiple reflections of the pump beam from the multilayer interfaces.

The synchronous behaviour of $N_{e h}$ and $\Gamma$ is in agreement with a conductivity model of a nondegenerate electron-hole gas in which the scattering rate is proportional to the carrier density. However, $N_{e h}$ and $\Gamma$ behave differently as a function of the pump wavelength. That is, $N_{e h}$ oscillates around a constant value, whereas $\Gamma$ tends to increase. This is because $N_{e h}$ depends only on the ab- 
sorbance, whereas $\Gamma$ has an additional dependence on the carrier temperature, which in turn depends on the excess energy provided by the pump photons. According to Eq. 2, a lower temperature (smaller photon energy) causes an increase in the scattering rate..

Finally, with the obtained $N_{e h}$ and $\Gamma$ values, we calculated the conductivity $\sigma_{0}$, as shown in Figure 6(c). Remarkably, the conductivity decreases monotonically as a function of the excitation energy. This result indicates that for the MB distribution, the nascent carrier conductivity simply depends on the excess energy (temperature) provided by the pump photons.

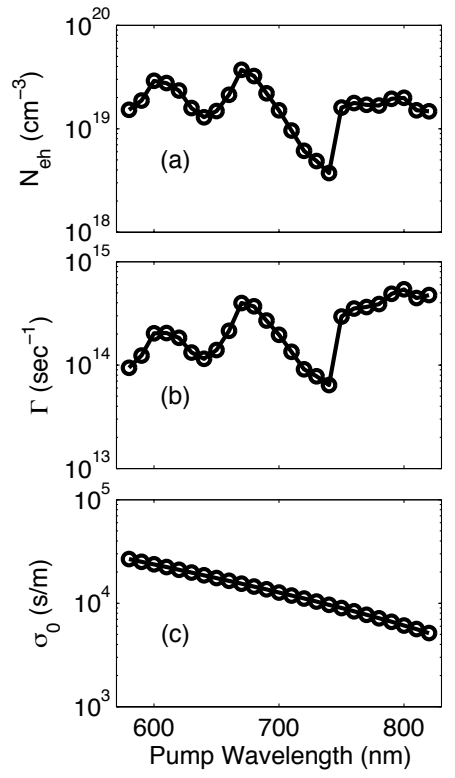

Figure 6: Excited carrier concentration $N_{e h}$, scattering rate $\Gamma$, and conductivity $\sigma_{0}$ as functions of pump center wavelength, obtained using Drude model and MB limitation fitting data $\Delta R / R_{0}$.

\section{Conclusion}

In conclusion, in this work we measured and analyzed the pump-probewavelength-dependent reflectivity of nc-Si:H samples at a fixed delay time of 
$300 \mathrm{fs}$. To establish the optical model of the samples, we investigated their composition with Raman and XRD methods and studied the optical properties using ellipsometry. To find the high-frequency conductivity at this short time, we simulated the pump-probe results using an optical model modified by the Drude contribution of the free carriers excited by the pump. The analysis revealed that the carrier thermodynamic distribution is better described by the classical MB statistics rather than the FD statistics. We found that the conductivity decreases monotonically as the excitation photon energy decreases, as expected for a classical electron-hole plasma. Our work shows that in the design of electro-optical devices one should carefully consider the effect of the balance between the carrier density and the excess energy in order to achieve a desired conductivity.

\section{Acknowledgments}

The Coherent laser system used in this research was obtained through the Birmingham Science City project: Creating and Characterising Next Generation Advanced Materials, supported by Advantage West Midlands (AWM) and funded in part by the European Regional Development Fund (ERDF). We thank T. Roger, J. Barreto, and D. Chekulaev for help with the experimental setup. We thank J. Bowen for the ellipsometry measurement. We thank EPSRC and DSTL for financial support.

\section{References}

[1] S. Sriraman, S. Agarwal, E. S. Aydil, D. Maroudas, Mechanism of hydrogen-induced crystallization of amorphous silicon, Nature 418 (6893) (2002) 62-65.

[2] A. Shah, P. Torres, R. Tscharner, N. Wyrsch, H. Keppner, Photovoltaic technology: the case for thin-film solar cells., Science 285 (1999) 692.

[3] A. Shah, J. Meier, E. Vallat-Sauvain, N. Wyrsch, U. Kroll, C. Droz, U. Graf, Material and solar cell research in microcrystalline silicon, Solar Energy Materials and Solar Cells 73 (1-4) (2003) 469-491. 
[4] S. Jung, J. Yi, Nanocrystalline-silicon thin-film nonvolatile memory devices for display applications, Electron Device Letters, IEEE 31 (9) (2010) 981983.

[5] C.-H. Lee, A. Sazonov, A. Nathan, J. Robertson, Directly deposited nanocrystalline silicon thin-film transistors with ultra high mobilities, Appl. Phys. Lett. 89 (2006) 252.

[6] A. Othonos, Applied physics reviews applied physics reviews probing ultrafast carrier and phonon dynamics in semiconductors, J. Appl. Phys. 83 (4) (1998) 1789.

[7] C. Shank, R. Yen, C. Hirlimann, Time-resolved reflectivity measurements of femtosecond-optical-pulse-induced phase transitions in silicon, Phys. Rev. Lett. 50 (1983) 454-457.

[8] A. Sabbah, D. M. Riffe, Femtosecond pump-probe reflectivity study of silicon carrier dynamics, Phys. Rev. B 66 (16) (2002) 165217.

[9] V. Freilikher, V. I. Tatarskii, M. Pustilnik, I. Yurkevich, Polarization of light scattered from slightly rough dielectric film, Optics Lett. 19 (18) (1994) 1382-1384.

[10] T. Hamasaki, H. Kurata, M. Hirose, Y. Osaka, Lowtemperature crystallization of doped asi:h alloys, Appl. Phys. Lett. 37 (12) (1980) 1084-1086.

[11] E. Bustarret, M. Hachicha, M. Brunel, Experimental determination of the nanocrystalline volume fraction in silicon thin films from raman spectroscopy, Appl. Phys. Lett. 52 (20) (1988) 1675-1677.

[12] O. Vetterl, F. Finger, R. Carius, P. Hapke, L. Houben, O. Kluth, A. Lambertz, A. Mück, B. Rech, H. Wagner, Intrinsic microcrystalline silicon: A new material for photovoltaics, Solar Energy Materials and Solar Cells 62 (1) (2000) 97-108. 
[13] H.P.King, L.E.Alexander, X-ray Diffraction Procedures, Wiley, New York, 1981.

[14] C. E. Bouldin, E. A. Stern, B. von Roedern, J. Azoulay, Structural study of hydrogenated a-ge using extended x-ray absorption fine structure, Phys. Rev. B 30 (1984) 4462-4469.

[15] L. Houben, M. Luysberg, P. Hapke, R. Carius, F. Finger, H. Wagner, Structural properties of microcrystalline silicon in the transition from highly crystalline to amorphous growth, Philosophical Magazine A 77 (6) (1998) $1447-1460$.

[16] P. Scherrer, Bestimmung der grösse und der inneren struktur von kolloidteilchen mittels röntgenstrahlen, Nachrichten von der Gesellschaft der Wissenschaften zu Göttingen, mathematisch-physikalische Klasse 1918 (1918) 98-100.

[17] A. Patterson, The scherrer formula for x-ray particle size determination, Phys. Rev. 56 (10) (1939) 978.

[18] A. Forouhi, I. Bloomer, Optical properties of crystalline semiconductors and dielectrics, Phys. Rev. B 38 (3) (1988) 1865.

[19] A. Forouhi, I. Bloomer, Optical dispersion relations for amorphous semiconductors and amorphous dielectrics, Phys. Rev. B 34 (10) (1986) 7018.

${ }_{285}$ [20] D. Zhang, E. Cherkaev, M. P. Lamoureux, Stieltjes representation of the 3d bruggeman effective medium and padé approximation, Applied Mathematics and Computation 217 (17) (2011) 7092-7107.

[21] E. Lioudakis, A. Othonos, G. Hadjisavvas, P. Kelires, A. Nassiopoulou, Quantum confinement and interface structure of si nanocrystals of sizes 35nm embedded in a-sio2, Physica E: Low-dimensional Systems and Nanostructures 38 (1) (2007) 128-134.

[22] E. Hecht, Optics, Pearson Education, 2012. 
[23] T. W. Roger, W. He, I. V. Yurkevich, A. Kaplan, Enhanced carrier-carrier interaction in optically pumped hydrogenated nanocrystalline silicon, Appl. Phys. Lett. 101 (14) (2012) 141904.

[24] A. Esser, H. Heesel, H. Kurz, C. Wang, G. Parsons, G. Lucovsky, Femtosecond spectroscopic study of ultrafast carrier relaxation in hydrogenated amorphous silicon a-si: H, J. Appl. Phys 73 (3) (1993) 1235-1239.

[25] K. Sokolowski-Tinten, D. Von der Linde, Generation of dense electron-hole plasmas in silicon, Phys. Rev. B 61 (4) (2000) 2643.

[26] W. He, I. V. Yurkevich, L. T. Canham, A. Loni, A. Kaplan, Determination of excitation profile and dielectric function spatial nonuniformity in porous silicon by using wkb approach, Opt. Express 22 (22) (2014) 27123-27135.

[27] A. Kaplan, A. Sajwani, Z. Li, R. Palmer, J. Wilcoxon, Efficient vacuum ultraviolet light frequency downconversion by thin films of cdse quantum dots, Appl. Phys. Lett. 88 (2006) 17105.

[28] J. Barreto, T. Roger, A. Kaplan, Resolving the ultrafast dynamics of charge carriers in nanocomposites, Appl. Phys. Lett. 100 (24) (2012) 241906.

[29] H. M. van Driel, Kinetics of high-density plasmas generated in si by 1060and $530 \mathrm{~nm}$ picosecond laser pulses, Phys. Rev. B 35 (15) (1987) 8166-8176.

[30] M. C. Downer, C. V. Shank, Ultrafast heating of silicon on sapphire by femtosecond optical pulses, Phys. Rev. Lett. 56 (1986) 761-764.

[31] M. Combescot, R. Combescot, Conductivity relaxation time due to electron-hole collisions in optically excited semiconductors, Phys. Rev. B 35 (1987) 7986-7992. 